\title{
Die vergewaltigte Frau: Was ist in der Notaufnahme zu tun?
}

\author{
Alexandra Ramshorn-Zimmer, Franziska Hilbig, Christian König, Romy Handzel, Michael Bernhard, \\ André Gries
}

\begin{abstract}
Die notfallmedizinische Versorgung von Opfern eines Sexualdelikts ist eine besondere Herausforderung: In einer aus Patientensicht bestehenden psychischen Ausnahmesituation müssen nicht nur Verletzungen versorgt, sondern auch eine gerichtsfeste Befunddokumentation und sachgerechte Spurensicherung gewährleistet werden.
\end{abstract}

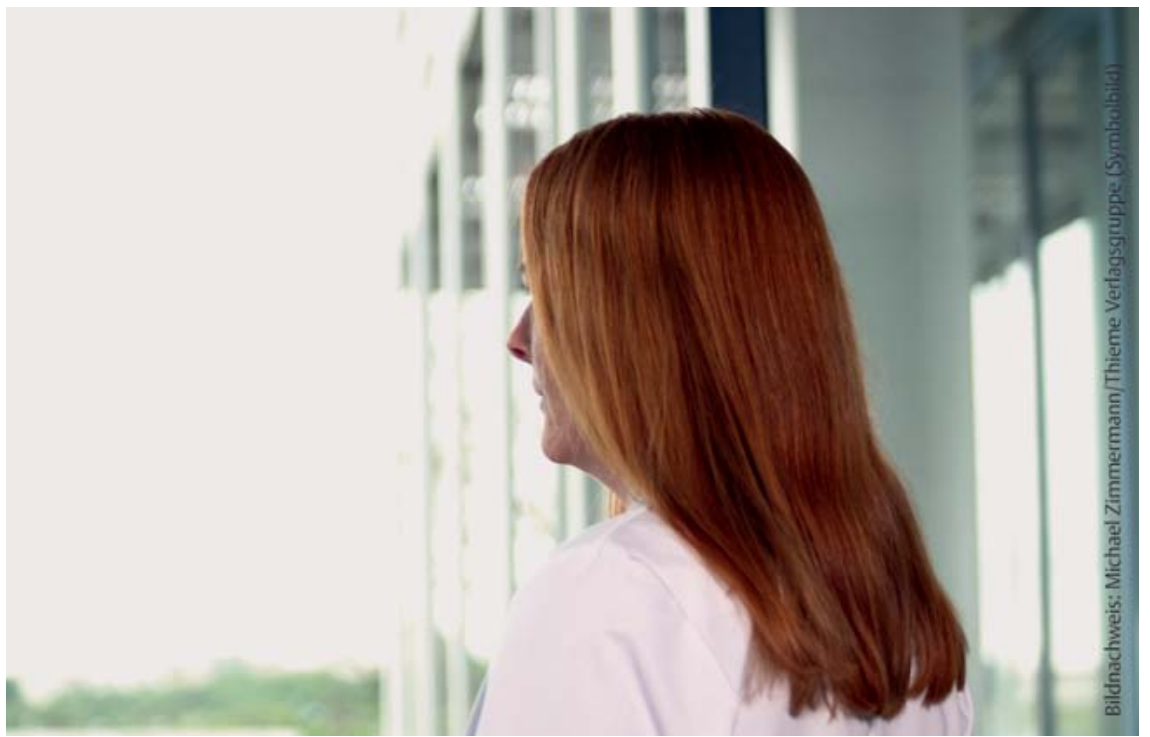

Die Versorgung eines Vergewaltigungsopfers in der Notaufnahme ist besonders anspruchsvoll: Fehlleistungen bei der Befunderhebung haben zwar in der Regel keine gravierenden gesundheitlichen Folgen. Allerdings können sie weitreichende juristische Konsequenzen zur Folge haben [1], da sich das gerichtliche Urteil letztlich auf die in der Notfallsituation erhobenen Beweismittel stützt.

Darüber hinaus sind die Möglichkeit der Übertragung von Infektionserkrankungen abzuwägen und entsprechende diagnostisch-therapeutische Maßnahmen in die Wege zu leiten. Gleiches gilt für die eventuelle Notwendigkeit einer Empfängnisverhütung. Im Sinne einer akuten Krisenintervention muss man zudem dafür sorgen, dass die Patientin psychologische Unterstützung erhält, und sie ggf. in eine sichere Unterbringung übergeben. Aufgrund der großen psychischen Belastung der Patientin sind Doppeluntersuchungen zu vermeiden - daher ist frühzeitig eine gynäkologische und rechtsmedizinische Beurteilung zu gewährleisten.

Der folgende Artikel soll dem (Notfall-) Mediziner Schritt für Schritt ein klinischforensisches Basiswissen vermitteln, das ihm die komplexe Versorgung dieser Patientinnen erleichtert und zur adäquaten Spurensicherung und fachgerechten Probenaufbewahrung beiträgt.

\section{Epidemiologie sexueller Straftaten $\nabla$}

Im Jahr 2012 wurden 8031 Vergewaltigungen bzw. sexuelle Nötigungen deutschlandweit zur Anzeige gebracht. Dabei waren 99\% der Beschuldigten männlichen und 96\% der Opfer weiblichen Geschlechts. Lediglich bei 21\% der Opfer bestand keine Vorbeziehung zum Täter. Eine Aufklärung der angezeigten Sexualverbrechen gelang in rund $80 \%$ der
Fälle [2]. Auch heute noch muss man von einer hohen Dunkelziffer ausgehen, da viele Opfer aus Scham, Angst oder einer persönlichen Beziehung zum Täter das Verbrechen nicht anzeigen. Bestimmte Bevölkerungsgruppen sind besonders gefährdet, z.B.:

- Behinderte

- Obdachlose

- Homo- und Transsexuelle

- Prostituierte [3]

Zudem nehmen Sexualstraftaten unter Alkohol- und Drogeneinfluss deutlich zu [4].

\section{Vorstellung in der zentralen Notaufnahme \\ $\nabla$}

\section{Medizinische Aspekte}

Neben den vom Rettungsdienst zugewiesenen Patientinnen stellen Notfallambulanzen häufig einen zentralen Anlaufpunkt für die eigeninitiierte Vorstellung von Opfern sexualisierter Gewalt dar. Sie suchen nicht nur medizinische Hilfe aufgrund physischer und psychischer Verletzungen, sondern brauchen Rat bez. möglicher Folgen: v.a. ungewollte Schwangerschaften oder potenziell übertragene infektiöse Erkrankungen. Obwohl die Ressourcen in der Notaufnahme häufig zeitlich und personell begrenzt sind, bedarf es hier der einfühlsamen Anamneseerhebung und anschließender Untersuchung.

\section{Rechtliche Aspekte}

Neben den medizinischen Aspekten muss man auch die rechtlichen Belange berücksichtigen: Die Patientin sollte darüber aufgeklärt werden, dass eine ausführliche Untersuchung in erster Linie dazu dient, die körperliche und seelische „Integrität“ 
wiederherzustellen. Andererseits ist sie jedoch auch Grundlage für eine vollumfängliche Beweissicherung als Basis für eine mögliche Anzeige. Die Untersuchung und Behandlung erfolgt in jedem Fall unabhängig von einer späteren Anzeige!

\section{Anamnese}

$\nabla$

Grundsätzlich muss man alle Einzelheiten der Tat erfragen und entsprechend dokumentieren (Infokasten). Bei polizeibeauftragten Untersuchungen gilt: Die Schilderung des Tathergangs durch die Polizei kann im Wissen, jedoch in Abwesenheit des Opfers erfolgen und als Grundlage für ergänzende anamnestische Fragestellungen dienen. Somit kann man zumindest teilweise vermeiden, das Opfer mit Mehrfachbefragungen zu belasten.

\section{Praxistipps:}

wichtige Anamnesefragen [1]

- Die 6 „Ws“ (Wer? Was? Wann? Wo? Wie viele? Welche Handlungen?)

- Gibt es akute Verletzungen? Bestehen Schmerzen oder Blutungen?

- Wie hat sich das Opfer nach der Tat verhalten? (Wechsel der Kleidung, Reinigung des Körpers)

- Hat das Opfer Medikamente, Drogen oder Alkohol vor oder zum Tatzeitpunkt konsumiert? Bestehen amnestische Defizite?

- Kam es täterseitig zu einer Ejakulation und wohin? Kam es zu sonstigen Sekretantragungen (z. B. Speichel)?

- Wann war die letzte Menstruationsblutung? Liegt eine Schwangerschaft vor? Wann und mit wem war der letzte einvernehmliche Geschlechtsverkehr? Befindet sich die Patientin in regelmäßiger gynäkologischer Behandlung?

- Wurden Verhütungsmittel verwendet?

\section{Die körperliche Untersuchung}

\section{$\boldsymbol{\nabla}$}

\section{Allgemeine Grundlagen}

Vorbereitung

Prinzipiell sollte man für eine ruhige Atmosphäre in einem separaten Untersuchungsraum sorgen und längere Wartezeiten vermeiden. Während der Unter- suchung sollte eine weibliche Drittperson anwesend sein, insbesondere wenn für die körperliche Untersuchung keine Ärztin verfügbar ist. Um das Gefühl des erneuten „Entblößens“ zu vermeiden, sollte bei der Ganzkörperuntersuchung schrittweise vorgegangen werden und lediglich die aktuell zu untersuchende Region entkleidet werden. Das Tragen von Handschuhen und Mundschutz ist zur Vermeidung einer Kontamination obligat. Nach Aufklärung über den Untersuchungszweck und -hergang muss man - die Einsichts- und Urteilsfähigkeit der Patientin vorausgesetzt - das Einverständnis zur Untersuchung, Probenentnahme, Sicherstellung und Weitergabe von Beweismitteln einholen und schriftlich dokumentieren.

\section{Anzeige}

Ist eine Anzeige beabsichtigt, gilt dies ebenso für die Schweigepflichtentbindung. Prinzipiell erfolgt die Untersuchung unabhängig von einer Anzeigenerstattung. Lehnt die Patientin eine Anzeigenerstattung $a b$, sollte man sie über die Möglichkeit einer vorsorglichen Asservierung von Spurenmaterial aufklären.

\section{Versorgung von Verletzungen}

Lebensbedrohliche Verletzungen müssen sofort behandelt werden. Dabei sollte man nach traumatologischen Standards zur Sicherung der Vitalfunktionen vorgehen (z.B. nach ATLS ${ }^{\circledR}$-Schema: Advanced Trauma Life Support). Wenn möglich sollte man die zu versorgenden Verletzungen vorab fotografisch dokumentieren (Übersichts- und Detailaufnahme mit Maßstab) [5]. Kleinere Verletzungen sind hingegen der rechtsmedizinischen Untersuchung hintanzustellen. Sollte es bei der Vergewaltigung zu traumatologisch relevanten Verletzungen gekommen sein, muss man eine entsprechende radiologische Diagnostik in die Wege leiten. Bei analer Penetration und damit assoziierter Schmerzsymptomatik ist ggf. eine Röntgen-Abdomen-Übersicht in Linksseitenlage zum Ausschluss einer Darmperforation zu ergänzen.

\section{Extragenitale Verletzungen Juristisch bedeutsam}

Bei mehr als zwei Drittel der Vergewaltigungsfälle kommt es zu extragenitalen Verletzungen; diese sind somit häufiger zu beobachten als genitale Traumata [6]. Aus juristischer Sicht ist der Nachweis derartiger Verletzungsmuster entscheidend, da diese meist zweifelsfrei eine Ge- waltanwendung belegen und dem oft täterseitig geäußerten Einwand widersprechen, alles sei freiwillig erfolgt.

\section{Verletzungsarten}

Vielfach kommt es zu stumpfer Gewalteinwirkung in Form von Schlägen. Typische Folgen von Vergewaltigungen sind zudem

- Fixierungsverletzungen ( $\bullet$ Abb. 1),

- passive und aktive Abwehrverletzungen v.a. im Bereich der Unterarme, Handrücken und Finger(-nägel),

- Widerlagerverletzungen ( $\bullet$ Abb. 2 ) und

- Verletzungen durch gewaltsames Entkleiden [1] ( $\bigcirc$ Abb. 3).

Eine Auflistung typischer extragenitaler Verletzungsmuster und ihrer jeweiligen Entstehungsmechanismen findet sich in O Tab. 1.

\section{Zu untersuchende Körperstellen}

Bei der ausführlichen körperlichen Untersuchung - im Sinne eines „secondary survey“ - ist darauf zu achten, dass insbesondere Verletzungen sowie Antragungen im Bereich verdeckter Körperstellen, wie z.B. der behaarten Kopfhaut, der Diagnostik nicht entgehen [1]. Sonst könnten folgende Verletzungen leicht übersehen werden:

- retroaurikuläre Verletzungen

- Lippen-/Mundschleimhautblutungen

- Hämatome und Hautabschürfungen im Bereich des Kehlkopfs, des Nackens und der behaarten Kopfhaut

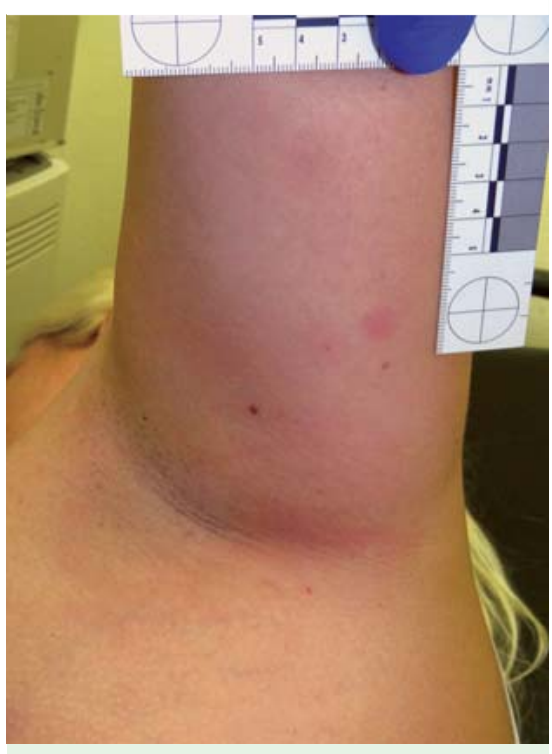

Abb. 1 Fixierungsverletzung: Griffhämatome i. B. der Oberarminnenseite (Bildnachweis: Institut für Rechtsmedizin Leipzig, Dr. C. König). 


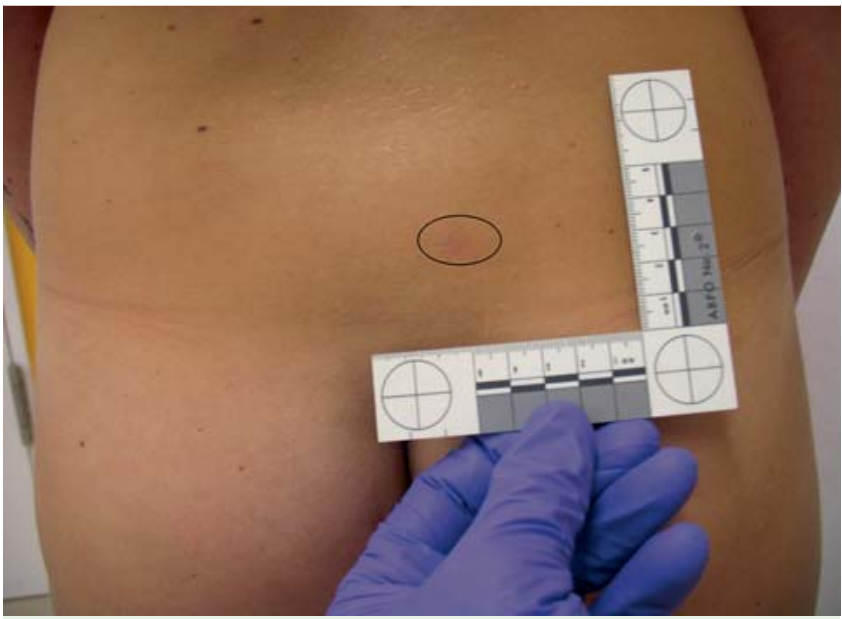

Abb. 2 Widerlagerverletzung im Bereich des Beckens (Bildnachweis: Institut für Rechtsmedizin Leipzig, Dr. C. König).

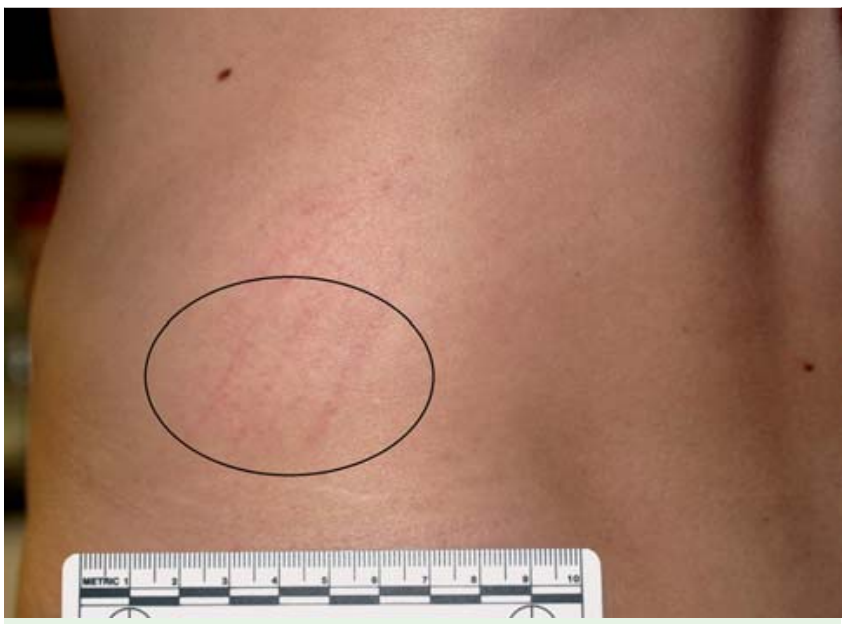

Abb. 3 Typische Kratzverletzungen nach gewaltsamem Entkleiden (Bildnachweis: Institut für Rechtsmedizin Leipzig, Dr. C. König).
Im Bereich des Kopfes sowie der Halsweichteile sollte man auf Hinweise für eine Strangulation, insbesondere konjunktivale Stauungsblutungen, achten. Im Rahmen der körperlichen Untersuchung sollten nicht nur Haut- und Weichteilverletzungen eruiert, sondern

- Frakturen,

- Verbrennungen und

- Verätzungen

aktiv ausgeschlossen werden [1].

\section{Anogenitale Untersuchung}

Anogenitale Verletzungen

Die anogenitale Untersuchung erfolgt idealerweise in Kooperation zwischen Rechtsmedizin und Gynäkologie, um für die Patientin belastende Doppeluntersuchungen zu vermeiden [1]. Verletzungen im Anogenitalbereich sind eher selten und präsentieren sich in Form geringer Rötungen, Hämatome oder oberflächlicher Schleimhautverletzungen ( $\bullet$ Abb. 4). Diese sind oft im Bereich der hinteren Kommissur der Labien lokalisiert [7]. Allerdings bedeutet das Fehlen derartiger Verletzungsmuster nicht, dass es zu keiner Gewaltanwendung und keiner vaginalen Penetration kam. Zunächst sollte man Abstriche der Oberschenkelinnenseiten sowie der Labien machen. Die Schamhaare müssen ggf. mit einem Einmalkamm ausgekämmt und mit dem Kamm in einem separaten Behältnis gesichert werden.

\section{Kolposkopie}

Neben der Inspektion sollte eine kolposkopische Diagnostik erfolgen, um die Schleimhautverhältnisse optimal zu beurteilen. Mit einem Spekulum untersucht

Tab. 1 Extragenitale Verletzungen mit Entstehungsmechanismus.

\begin{tabular}{|c|c|c|}
\hline Lokalisation & Art der Verletzungen & Entstehungsmechanismus \\
\hline \multirow[t]{4}{*}{ Kopf } & punktförmige Rötungen der Kopfhaut & Ausreißen von Haaren \\
\hline & petechiale Blutungen in Haut und Schleimhäuten & $\begin{array}{l}\text { Stauungsblutungen nach } \\
\text { Angriff gegen den Hals }\end{array}$ \\
\hline & Hämatome an Wangen, Ohren, retroaurikulär & Schläge \\
\hline & $\begin{array}{l}\text { Hämatome und Verletzungen der Mundvorhof- } \\
\text { schleimhaut }\end{array}$ & $\begin{array}{l}\text { Schläge, gewaltsamer } \\
\text { Verschluss des Mundes }\end{array}$ \\
\hline Hals & $\begin{array}{l}\text { Rötungen, kratzerartige Hautabschürfungen, } \\
\text { halbmondförmige Einblutungen, Oberhautverlet- } \\
\text { zungen (Halsschmerzen, Schluckbeschwerden) }\end{array}$ & $\begin{array}{l}\text { Angriff gegen den Hals } \\
\text { (Würgen, Drosseln) }\end{array}$ \\
\hline $\begin{array}{l}\text { Rumpf- } \\
\text { vorderseite }\end{array}$ & Hämatome, insbesondere der Mammae & $\begin{array}{l}\text { Bissverletzungen, Saugbisse, } \\
\text { Griffhämatome }\end{array}$ \\
\hline $\begin{array}{l}\text { Rumpf- } \\
\text { rückseite }\end{array}$ & $\begin{array}{l}\text { flüchtige Rötungen, Unterblutungen, z. T. Exkoria- } \\
\text { tionen über prominenten Knochenpunkten } \\
\text { (Schulterblätter, Dornfortsätze der Brust- und } \\
\text { Lendenwirbelsäule, Kreuzbein) }\end{array}$ & Widerlagerverletzungen \\
\hline $\begin{array}{l}\text { Becken- und } \\
\text { Gesäßregion }\end{array}$ & $\begin{array}{l}\text { streifenförmige Exkoriationen und } \\
\text { Kratzverletzungen, senkrecht verlaufend }\end{array}$ & Entkleideverletzungen \\
\hline \multirow[t]{4}{*}{ Arme } & $\begin{array}{l}\text { Rötungen, Hämatome, z. T. Kratzverletzungen } \\
\text { und flächige Exkoriationen an Oberarmen }\end{array}$ & $\begin{array}{l}\text { Griffhämatome, } \\
\text { Fixierverletzungen }\end{array}$ \\
\hline & $\begin{array}{l}\text { Hämatome, Schwellungen an Unterarmen } \\
\text { (bes. ulnar) und Handrücken }\end{array}$ & Abdeck-/Parierverletzungen \\
\hline & $\begin{array}{l}\text { quer verlaufende, streifige Druckmarken, } \\
\text { Hämatome, Exkoriationen im Handgelenkbereich }\end{array}$ & Fesselung \\
\hline & $\begin{array}{l}\text { glattrandige Verletzungen an Händen } \\
\text { (bes. Handflächen) }\end{array}$ & $\begin{array}{l}\text { Abwehr gegen Angriff } \\
\text { mit scharfem Werkzeug }\end{array}$ \\
\hline \multirow[t]{2}{*}{ Beine } & $\begin{array}{l}\text { Rötungen, Hämatome, Kratzverletzungen } \\
\text { besonders an den Oberschenkeln innenseitig }\end{array}$ & $\begin{array}{l}\text { Spreizverletzungen, } \\
\text { Entkleideverletzungen }\end{array}$ \\
\hline & doppelstreifig angeordnete Unterblutungen & Schläge mit Stock o. ä. \\
\hline
\end{tabular}

man die inneren Genitalien auf Verletzungen, Sekretansammlungen, frische Blutungen und Fremdkörper. Hierbei sollten mindestens 2 Abstriche im Bereich des Scheideneingangs und des Scheidengewölbes abgenommen werden. Diese sind durch Abstriche im Bereich der Portio zu ergänzen.

\section{Ultraschall}

Um innere Blutungen auszuschließen, vervollständigt eine Untersuchung mittels transvaginalem/abdominellem Ultraschall die gynäkologische Diagnostik [1]. 


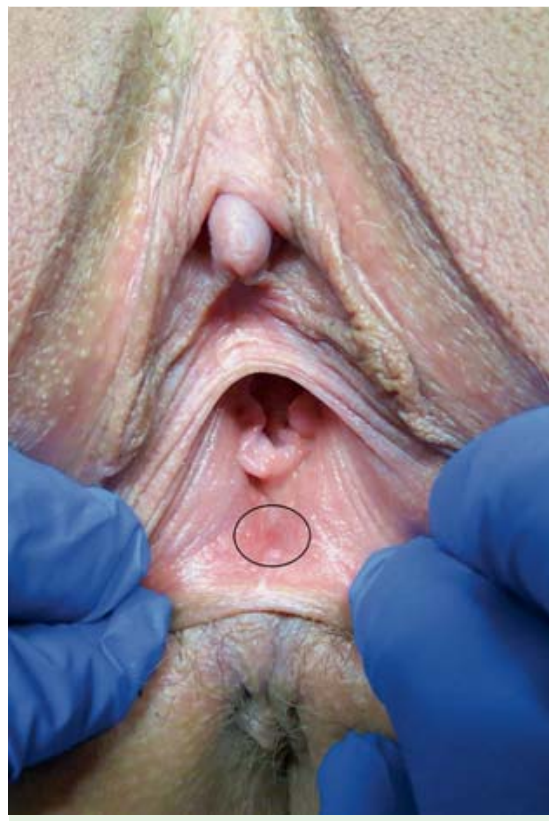

Abb. 4 Typisches genitales Verletzungsmuster mit diskreter Rötung i. B. der hinteren Scheidenkommissur (Bildnachweis: Institut für Rechtsmedizin Leipzig, Dr. C. König).

\section{Perianalregion}

Nachdem die Perianalregion inspiziert wurde, sollte man Abstriche im Bereich der Afteröffnung und aus dem Anus entnehmen. Bei peranalen Blutabgängen sollte man ggf. eine proktologische Untersuchung ergänzen.

\section{Befund- und Spurensicherung $\nabla$}

\section{Rechtsmedizin}

Wenn verfügbar, sollte man zügig zur Erstversorgung konsiliarisch einen Rechtsmediziner für die Dokumentation und Interpretation der Verletzungen sowie die Sicherung von weiteren Spuren heranziehen. Ist kein Rechtsmediziner verfügbar, kann man ggf. ein rechtsmedizinisches Telekonsil einholen. Ursprünglich vom Leipziger Institut für Rechtsmedizin entwickelt, werden diese mittlerweile von mehreren rechtsmedizinischen Instituten in Deutschland angeboten [8].

\section{Entnahme von Proben}

Biologische Spuren am Körper sollte man gezielt und nur dort asservieren, wo Sekretspuren erkennbar sind oder wo man sie aufgrund der Opferangaben vermutet. Man sollte die Haut nicht großflächig mit Asservatentupfern abreiben, da so v.a. die DNA des Opfers erfasst wird [9]. Die Probenentnahme sollte - wenn möglich - in-

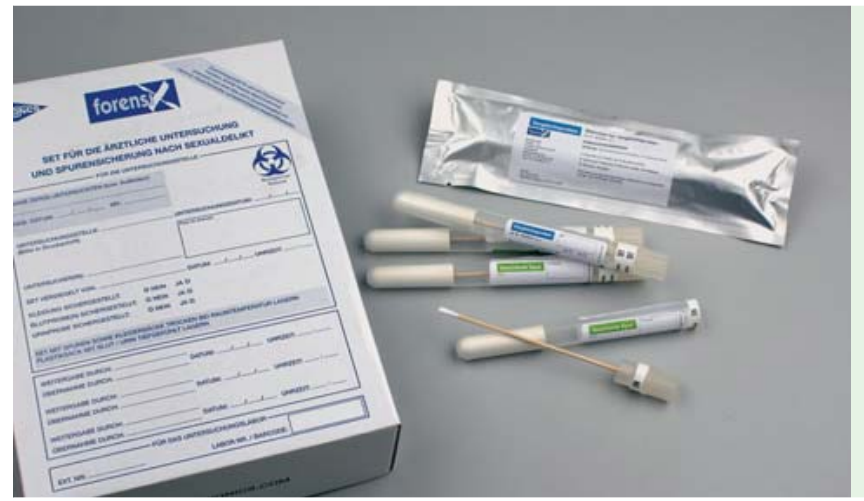

Abb. 5 Spurensicherungsset (Bildnachweis: Institut für Rechtsmedizin Leipzig, Dr. C. König).

nerhalb von $72 \mathrm{~h}$ nach der Tat erfolgen. Um sie genau zuordnen zu können, sollte man die Befunde und Proben korrekt identifizieren: mit Dokumentation von Personalien des Patienten, Datum und Lokalisation der Abstrichpräparate [1].

\section{Lagerung von Proben}

Die kontaminationsfreie Entnahme von Abstrichen erfolgt bei trockenen Substanzen mit einem sterilen, befeuchteten Wattestieltupfer, der im Anschluss getrocknet werden muss. Eine feuchte Lagerung führt häufig zur Zerstörung der Proben und verhindert eine verwertbare DNA-Analyse. Feuchtes Spurenmaterial wird mit einem trockenen Tupfer aufgenommen und anschließend getrocknet. Für solche Zwecke gibt es kommerziell angebotene Spurensicherungsbestecke ( $\boldsymbol{A}$ Abb. 5). Diese Spurensicherungssets und standardisierte Checklisten gewährleisten

- die Erhebung aller relevanten Untersuchungsbefunde,

- die Dokumentation der Verletzungen und

- die sachgerechte Spurensicherung und -aufbewahrung $[9,10]$.

Eine umfangreiche Sammlung entsprechender Dokumentationsbögen findet sich unter den im Infokasten aufgeführten Internetlinks.

\section{Blut- und Urinprobe}

Grundsätzlich empfiehlt sich eine Blutund Urinasservierung zum Nachweis bzw. Ausschluss eines Betäubungsmittel-, Medikamenten- oder Alkoholkonsums. Eine Haarprobe kann ggf. zusätzlich gesichert werden. Falls das Opfer eine unerklärte Bewusstlosigkeit oder Gedächtnislücken schildert, muss man an die Möglichkeit einer unwissentlichen Verabreichung von kurzwirksamen Narkotika wie z.B. $\gamma$-Hydroxybutyrat denken. Da diese Substanzen z.T. sehr schnell im Blut abgebaut werden, sollte die Blutentnahme und Urinasservierung so schnell wie möglich erfolgen [9].

\section{Schwangerschaft \\ $\nabla$}

Um eine vorbestehende Schwangerschaft auszuschließen, sollte die Analyse von $\beta$ HCG im Serum erfolgen. Das Risiko einer vergewaltigungsassoziierten Schwangerschaft beträgt $5 \%$ [11]. Auf Wunsch der Patientin kann eine Notfallkontrazeption mit Levonorgestrel (z.B. 1-mal PiDaNa ${ }^{\circledR}$ $1,5 \mathrm{mg}$ p.o.) innerhalb von $72 \mathrm{~h}$ bzw. Ulipristal (1-mal EllaOne ${ }^{\circledR} 30 \mathrm{mg}$ ) innerhalb von $5 \mathrm{~d}$ nach Geschlechtsverkehr initiiert werden. In diesem Zusammenhang muss man die Patientin über arzneimittelassoziierte Risiken und Nebenwirkungen wie z.B. Übelkeit und Erbrechen, abdominelle Schmerzen und vaginalen Blutabgang aufklären. Im Falle einer schon vor dem Delikt bestehenden Schwangerschaft sind regelmäßige gynäkologische Verlaufskontrollen zu empfehlen.

\section{Übertragung infektiöser Erkrankungen \\ $\nabla$}

\section{Wann untersuchen?}

Nach einem Sexualdelikt muss man die Patientin nicht routinemäßig auf alle sexuell übertragbaren Krankheiten untersuchen. Liegen jedoch ein entsprechender Verdacht und klinische Befunde vor, sollten serologische/mikrobiologische Untersuchungen durchgeführt werden: So kann eine vorbestehende Infektionserkrankung ausgeschlossen und eine durch den Übergriff übertragene nachgewiesen werden (@ Tab.2). Außerdem muss die Patientin darauf hingewiesen werden, dass Verlaufsuntersuchungen entsprechend den virologisch/mikrobiologischen Standards zur Diagnostik von Infektionserkrankun- 
Tab. 2 Sexuell übertragbare Infektionserkrankungen.

\begin{tabular}{|c|c|}
\hline $\begin{array}{l}\text { Infektions- } \\
\text { erkrankung }\end{array}$ & $\begin{array}{l}\text { diagnostische } \\
\text { Maßnahmen }\end{array}$ \\
\hline $\begin{array}{l}\text { Humanes Immun- } \\
\text { defizienz-Virus (HIV) }\end{array}$ & Serologie \\
\hline Hepatitis B und C & Serologie, PCR \\
\hline Syphilis & Serologie \\
\hline Gonorrhö & Kultur, PCR \\
\hline Chlamydien & Kultur, PCR \\
\hline Trichomonaden & Nativausstrich, Kultur \\
\hline
\end{tabular}

gen notwendig sind. Wie bei anderen Verletzungen auch üblich, sollte man den aktuellen Tetanusimpfstatus klären.

\section{Prophylaxe}

Bei unzureichender Immunisierung sollte man im Anschluss eine Vakzinierung gegen Hepatitis B und Tetanus mit der Patientin besprechen und durchführen. Auch eine Postexpositionsprophylaxe (PEP) muss man ggf. zu diesem Zeitpunkt vornehmen. Dabei sind zu berücksichtigen:

- ggf. bekannte HIV-Infektion eines bekannten Täters

- Verwendung von Verhütungsmitteln

- lokale HIV-Prävalenzen bei unbekanntem Täter

- Risiko der Transmission in Abhängigkeit von der Art der Penetration (HIVTransmissions-Risiko bei analer Penetration: 1-3 Fälle/100 Patienten, bei vaginaler Penetration: 1-2 Fälle/1000 Patienten [12])

Die Patientin sollte man darauf hinweisen, dass unmittelbar mit der Behandlung begonnen werden muss - die Wirksamkeit der PEP hängt maßgeblich vom Zeit- raum zwischen Exposition und Therapiebeginn ab. Der erstbehandelnde Arzt stellt bei entsprechender Risikokonstellation die Indikation zur PEP. Ausführliche Informationen zur Indikationsstellung, Durchführung, Nebenwirkungsprofil und versicherungsrechtlichen Aspekten sind den Internetseiten des Robert Koch-Instituts sowie der deutschen AIDS-Gesellschaft e.V. (Infokasten) zu entnehmen.

\section{Dokumentation}

Die Beratung sowie die Einverständniserklärung zur PEP müssen dokumentiert werden. Entsprechende Formulare, die das Robert Koch-Institut zur Verfügung stellt, finden sich ebenfalls als Internetlinks im Infokasten.

\section{Psychische Traumatisierung \\ $\nabla$}

\section{Frühzeitige Hilfe}

Neben den physischen Verletzungen kommt es bei einem überwiegenden Teil der Patientinnen zu einer psychischen Traumatisierung [13]. Die entsprechenden Befunde muss man sorgfältig dokumentieren. Es ist sinnvoll, frühzeitig psychosoziale Hilfe zu veranlassen, um Folgeschäden zu vermeiden bzw. zu minimieren. Dem behandelnden Arzt sollten regionale Beratungs-und Hilfsangebote bekannt sein. Regionale und überregionale Hilfsangebote sind u.a.:

- Autonomes Frauenhaus Leipzig, Tel.: $0341 / 4798179$

- Frauennotruf Leipzig, Tel.: 0341/ 3911199, http://www.frauennotrufleipzig.de

- Bundesweites „Hilfstelefon Gewalt gegen Frauen“: 0800/116016

- Weißer Ring, Opfernotruf \& Info-Telefon (24h): $01803 / 343434$

Im Internet

- Dokumentationsbögen zur systematischen Untersuchung und Befundsicherung: http://www.frauennotruf-frankfurt.de/Aerztliche-Dokumentation.40.0.html

- Umfangreiche Informationen zur Indikation, Durchführung, Nebenwirkungen und Behandlungskosten der PEP: http://www.daignet.de

- Robert Koch-Institut: HIV/AIDS-Ratgeber für Ärzte: http://www.rki.de/DE/Content/ Infekt/EpidBull/Merkblaetter/Ratgeber_HIV_AIDS.html

- Robert Koch-Institut: Mustervorlage zur Dokumentation der PEP einer HIV-Infektion: http://www.rki.de/DE/Content/InfAZ/H/HIVAIDS/PEP_Dokumentationsbogen_2008. html

- Nützliche Hinweise für Opfer sexualisierter Gewalt: http://www.gewaltgegenfrauen. de/beratung-und-hilfe/vergewaltigung.htm

- Pro Familia e. V.: örtliche Anschrift und Sprechzeiten unter http://www.Frauen-gegenGewalt.de

\section{Weitere Versorgung}

Gibt es Hinweise auf eine vorliegende Eigengefährdung, sollte man unmittelbar eine fachpsychiatrische Mitbeurteilung und ggf. stationäre Behandlung veranlassen. Bei Entlassung aus der Klinik muss ausgeschlossen sein, dass die Patientin in eine erneute unmittelbare Gefährdungssituation gerät. Ist dies nicht gewährleistet, ist die stationäre Aufnahme oder Unterbringung in einer entsprechenden Schutzeinrichtung indiziert. Hierbei sollten die behandelnden Ärzte kooperativ mit den zuständigen Sozialdiensten und den lokalen Sicherheitsbehörden zusammenarbeiten.

\section{Medikolegale Aspekte}

$\nabla$

\section{Schweigepflicht}

Bei patientenbeauftragter Untersuchung gilt prinzipiell die ärztliche Schweigepflicht. Nach der Untersuchung erhält die Patientin eine Bescheinigung über Ort und Zeit der Untersuchung sowie über die erhobenen Asservate; diese kann ggf. den Ermittlungsbehörden vorgelegt werden. Wenn die Patientin zur polizeibeauftragten Untersuchung kommt, ist der Arzt durch die polizeilich durchzuführende Schweigepflichtentbindung von der selbigen befreit. Ist die Patientin zur Aussageverweigerung berechtigt, z.B. wenn es sich bei dem Täter um einen Familienangehörigen handelt, darf die Entbindung von der Schweigepflicht zurückgenommen werden [1].

\section{Aufbewahrung der Beweise}

In Abhängigkeit regionaler Gegebenheiten kann das erhobene Beweismaterial an das zuständige Institut für Rechtsmedizin weitergeleitet und dort für eine bestimmte Frist aufbewahrt werden. Die Geschädigte muss sich somit nicht unmittelbar für oder gegen eine Strafanzeige entscheiden. Erst nach Beauftragung durch die Staatsanwaltschaft wird das Beweismaterial analysiert.

\section{Fazit}

$\nabla$

Die Versorgung von Opfern sexualisierter Gewalt ist eine komplexe Aufgabe, die sowohl medizinische, psychologische als auch rechtliche Aspekte beinhaltet. Eine interdisziplinäre Zusammenarbeit in der zentralen Notaufnahme und ein abgestimmtes Vorgehen sind Grundlage für eine optimale Patientenversorgung und 
Checkliste zur Versorgung von Vergewaltigungsopfern [1]

1. Erstkontakt Patient-Arzt

2. Anamneseerhebung (gynäkologische Anamnese, Zyklus, Kontrazeption, letzter einvernehmlicher Geschlechtsverkehr)

3. Untersuchung

1. Schritt: Abstriche oral

2. Schritt: Sicherstellen der Kleidung (Papiersack)

- 3. Schritt: körperliche Untersuchung mit Spurensicherung und Dokumentation auf Untersuchungsbögen

- 4. Schritt: Blut- und Urinproben

- 5. Schritt: gynäkologische Untersuchung und Spurensicherung

- 6. Schritt: Abstriche Anus/Rektum

- 7. Schritt: weiterführende Diagnostik (bildgebende Verfahren etc.)

4. Klinische Behandlungsmaßnahmen: Versorgung behandlungsbedürftiger Verletzungen, Verabreichung bzw. Verschreibung von Antikonzeptiva, Impfung sowie medikamentöse Therapie inkl. Postexpositionsprophylaxe (PEP) bei Möglichkeit der Übertragung von infektiösen Krankheiten

5. Psychosoziale Betreuung der Opfer mit Verweis auf spezifische Beratungsstellen und Betreuungseinrichtungen

die Sicherung vorhandener Spuren, die einen wichtigen Aspekt bei der juristischen Beurteilung darstellen. Die Verwendung von Checklisten und Spurensicherungssets sowie regelmäßige Schulungsmaßnahmen können die Versorgung von Opfern sexueller Gewalt erleichtern und optimieren.

\section{Hinweis}

Erstpublikation in: Lege Artis 2014; 4: 250-256 senkonflikte vorliegen.

\section{Literatur} 2013 ministerium des Inneren
4 Lawyer S, Resnick H, Bakanic V et al. Forcible, drug-facilitated and incapacitated rape and sexual assault among undergraduate women. J Am Coll Health 2010; 58: 453-460

5 Verhoff MA, Gehl A, Kettner $M$ et al. Digitale forensische Fotodokumentation. Rechtsmedizin 2009; 19: 369-381

6 Kernbach G, Püschel K, Brinkmann B. Extragenitale Verletzungen bei Vergewaltigung. Geburtsh Frauenheilk 1984; 44: 643-650

7 Sommers MS. Defining patterns of genital injury from sexual assault: a review. Trauma Violence Abuse 2007; 8: 270-280

8 Hädrich C. Forensische Aspekte der Notfallmedizin. Intensiv- und Notfallbehandlung 2013; 38: 1-10

9 Banaschak S, Gerlach K, Seifert D et al. Forensisch-medizinische Untersuchung von $\mathrm{Ge}$ waltopfern - Empfehlungen der Deutschen Gesellschaft für Rechtsmedizin auf der Grundlage der Empfehlungen der Schweizer Gesellschaft für Rechtsmedizin. Rechtsmedizin 2011; 21: 483-488

10 Parnis D, Du Mont J. Examining the standardized application of rape kits: an exploratory study of post post-sexual assault professional practices. Health Care Women Int 2002; 23: 846-853

11 Holmes MM, Resnick HS, Kolpatrick DG et al. Rape-related pregnancy: estimates and descriptive characteristics from a national sample of women. Am J Obstet Gynecol 1996; 175: 320-324

Die Autoren erklären, dass keine Interes-

1 Grassberger M, Türk E, Yen K. Klinisch-forensische Medizin. Berlin, Heidelberg: Springer;

2 Polizeiliche Kriminalstatistik 2012, Bundes-

3 Acierno R, Resnick H, Kolpatrick DG et al. Risk factors for rape, physical assault and posttraumatic stress disorder in women: examination of differential multivariate relationships. J Anxiety Disord 1999; 13: 541-563
2 Boily MC, Baggaley RF, Wang $L$ et al. Heterosexual risk of HIV-1 infection per sexual act: systematic review and meta-analysis of observational studies. Lancet Infet Dis 2009; 9: $118-129$

13 Linden JA. Clinical practice. Care of the adult patient after sexual assault. N Engl J Med 2011; 365: 834-841

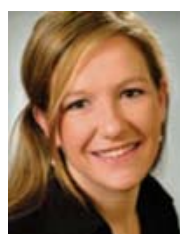

Korrespondenz

Dr. med. Alexandra Ramshorn-Zimmer

Zentrale Notaufnahme des Universitätsklinikums Leipzig, Standort Mitte der Stadt Leipzig

alexandra.ramshorn-zimmer@ medizin.uni-leipzig.de 